\title{
Total public announcements
}

\author{
David Steiner*† Thomas Studer ${ }^{\dagger}$
}

February 2007

\begin{abstract}
We present a dynamic epistemic logic for knowledge change of rational agents. Existing approaches only deal with partial public announcements, that means an announcement may lead to an inconsistent state. We introduce an extension of the multi-modal logic $\mathrm{S} 5_{n}$ featuring total public announcements where an update cannot result in an inconsistency. We also study total public announcements in the context of common knowledge and relativized common knowledge.
\end{abstract}

\section{Introduction}

At the end of the eighties, Plaza published the famous article about logics of public communications [13]. In the sequel, the theory of knowledge change caused by incoming information has been further developed by many authors. We confine ourselves to mentioning just a few typical articles: Baltag et al. [1, 2], van Benthem et al. [3, 4, 5, 6], van Ditmarsch et al. [7, 8, 9, 10], as well as Renne [14].

The language for logics of public announcements is the language of standard multi-modal logic augmented with announcement operators $[\alpha]$ for every formula $\alpha$. The expression $[\alpha] \beta$ then stands for after every announcement of $\alpha$, it holds that $\beta$. In the classical setting, announcement has to be read as truthful announcement. Therefore, announcements are partial: that is

$$
\neg[\alpha] \perp
$$

is not valid (see [9], Proposition 4.11).

\footnotetext{
${ }^{*}$ Research partly supported by the Swiss National Science Foundation

${ }^{\dagger}$ Institut für Informatik und angewandte Mathematik, Universität Bern, Neubrückstrasse 10, CH-3012 Bern, Switzerland. Email: \{steiner, tstuder\}@iam.unibe.ch
} 
We propose a system in which announcements are total, that is (1) holds. Therefore, in our system announcements need not be truthful; they can be true or false. As usual, a true announcement will lead to an update of an agent's epistemic state. However, a false announcement will not lead to an inconsistent epistemic state, it will automatically be ignored by the agents. That is, after a false announcement, an agent will have the same epistemic state as before the announcement. Because (1) holds in our system, we call it consistency preserving. A system for consistency preserving belief change (instead of knowledge change) is studied in [15].

A property we keep from the classical setting is

$$
p \rightarrow[\alpha] p
$$

That means, an announcement does not change atomic facts. We call a system that satisfies (2) atomic preserving.

The paper is organized as follows. In Section 2, we present an axiomatization of a system for total public announcements which satisfies both (1) and (2). We also propose a Kripke semantics for our system and show soundness and completeness of our axiomatization. In Section 3, we extend our logic with common knowledge operators. We show that agents can achieve common knowledge by receiving an announcement. For this system, the completeness proof cannot make use of a translation to a language without announcements (which is possible in the case without common knowledge). Completeness of total public announcements with common knowledge is established via the notion of maximal consistent sets. Recently, relativized common knowledge has received much attention in the context of logics for public announcements $[6,4,5]$. We introduce a system of relativized common knowledge and total public announcements in Section 4. Again, we establish soundness and completeness of our system. It follows from the proof that the addition of total public announcements to relativized common knowledge does not increase the expressive power of the language. We conclude this paper with some discussion in Section 5.

\section{A system for total public announcements}

We introduce the language $\mathcal{L}_{n}^{\mathrm{A}}$ of multi-modal logic with dynamic-style operators for public announcements.

Given a natural number $n \geq 1$, we fix the set $\mathcal{A}=\{1, \ldots, n\}$ of $n$ rational agents. Further, we take a countable non-empty set $\mathcal{P}$ of propositions, denoted by $p, q, \ldots$, possibly with subscripts. The set of $\mathcal{L}_{n}^{\mathrm{A}}$ formulas is defined 
by the following grammar $(p \in \mathcal{P}, i \in \mathcal{A})$,

$$
\alpha, \beta, \ldots::=p|\neg \alpha| \alpha \wedge \beta\left|K_{i} \alpha\right|[\alpha] \beta .
$$

The formula $K_{i} \alpha$ stands for the agent $i$ knows $\alpha$, the formula $[\alpha] \beta$ means $\beta$ holds after the public announcement of $\alpha$. The $\mathcal{L}_{0}$ formulas are the propositional formulas, the $\mathcal{L}_{n}$ formulas are the modal formulas without announcement operators.

For all formulas $\alpha, \beta$ of $\mathcal{L}_{n}^{\mathrm{A}}$, we define $\alpha \vee \beta, \alpha \rightarrow \beta$, and $\alpha \leftrightarrow \beta$ as usual. Further, we let

$$
\top:=p_{0} \vee \neg p_{0} \quad \text { and } \quad \perp:=p_{0} \wedge \neg p_{0}
$$

for some fixed $p_{0} \in \mathcal{P}$. Iterated announcements $[\alpha]^{k} \beta$ are defined by induction on $k$. We set

$$
[\alpha]^{0} \beta:=\beta \quad \text { and } \quad[\alpha]^{k+1} \beta:=[\alpha][\alpha]^{k} \beta .
$$

The following system for total public announcements in the context of belief change can be obtained from Gerbrandy and Groeneveld [12]. It results from deleting edges in a Kripke structure.

(PT) Every instance of a propositional tautology,

(K) $\quad K_{i}(\alpha \rightarrow \beta) \rightarrow\left(K_{i} \alpha \rightarrow K_{i} \beta\right)$,

(4) $\quad K_{i} \alpha \rightarrow K_{i} K_{i} \alpha$,

(5) $\quad \neg K_{i} \alpha \rightarrow K_{i} \neg K_{i} \alpha$,

(A1) $\quad[\alpha] p \leftrightarrow p$,

$(\mathrm{A} 2) \quad[\alpha](\beta \rightarrow \gamma) \rightarrow([\alpha] \beta \rightarrow[\alpha] \gamma)$,

(A3) $\quad[\alpha] \neg \beta \leftrightarrow \neg[\alpha] \beta$,

(A4.B) $\quad[\alpha] K_{i} \beta \leftrightarrow K_{i}(\alpha \rightarrow[\alpha] \beta)$,

$$
(\mathrm{MP}) \frac{\alpha \quad \alpha \rightarrow \beta}{\beta}, \quad(\text { NEC. } 1) \frac{\alpha}{K_{i} \alpha}, \quad \text { (NEC.2) } \frac{\alpha}{[\beta] \alpha} .
$$

To obtain a system for knowledge change, we cannot just add the knowledge axiom $K_{i} \alpha \rightarrow \alpha$ since that would lead to an inconsistency. The theory $\mathrm{S}_{n}^{\mathrm{A}}$ is obtained by changing axiom (A4.B) to (A4) and adding axioms (T) and (A5).

( T) $\quad K_{i} \alpha \rightarrow \alpha$,

(A4) $\quad \alpha \rightarrow\left([\alpha] K_{i} \beta \leftrightarrow K_{i}(\alpha \rightarrow[\alpha] \beta)\right)$,

(A5) $\quad \neg \alpha \rightarrow([\alpha] \beta \leftrightarrow \beta)$. 
The instances of axiom (A5) of the form

$$
\neg \alpha \rightarrow\left([\alpha] K_{i} \beta \leftrightarrow K_{i} \beta\right)
$$

can be seen as a necessary companion to the knowledge axiom ( $T$ ), because a false formula can never be known by an agent, thus it can never be learned. Note that we could formulate the system $\mathrm{S} 5_{n}^{\mathrm{A}}$ with (3) instead of (A5). Then (A5) would be provable in the resulting system. However, later we will consider an extension of $\mathrm{S}_{n}^{\mathrm{A}}$ by common knowledge operators. There, things get much simpler if (A5) is already included as an axiom.

By replacing ( $\mathrm{T}$ ) with the (D)-axiom $\neg K_{i} \perp$, the agents can decide whether to accept an incoming formula or to reject it. For a detailed treatment of this approach, see [15].

Our system is consistency preserving as well as atomic preserving. We have the following lemma.

Lemma 1 For all $\mathcal{L}_{n}^{\mathrm{A}}$ formulas $\alpha$ and all propositions $p \in P$ we have

$$
\mathrm{S} 5_{n}^{\mathrm{A}} \vdash \neg[\alpha] \perp \quad \text { and } \quad \mathrm{S}_{n}^{\mathrm{A}} \vdash p \rightarrow[\alpha] p .
$$

Observe, that the public announcement operator is self-dual due to axiom (A3). This means we do not have to distinct the statements ' $\beta$ holds after every (truthful) public announcement of $\alpha$ ' and ' $\beta$ holds after some (truthful) public announcement of $\alpha$ '. In our setting, there is only one public announcement of a formula. It can be truthful or not.

We will now state some properties which will be helpful for the rest of the paper.

Lemma 2 For all $\mathcal{L}_{n}^{\mathrm{A}}$ formulas $\alpha, \beta$, and $\gamma$ we have that $\mathrm{S} 5_{n}^{\mathrm{A}}$ proves

$$
\begin{aligned}
{[\alpha](\beta \wedge \gamma) } & \leftrightarrow([\alpha] \beta \wedge[\alpha] \gamma) \\
{[\alpha](\beta \vee \gamma) } & \leftrightarrow([\alpha] \beta \vee[\alpha] \gamma) \\
{[\alpha] K_{i} \beta } & \leftrightarrow\left(\neg \alpha \wedge K_{i} \beta\right) \vee\left(\alpha \wedge K_{i}(\alpha \rightarrow[\alpha] \beta)\right)
\end{aligned}
$$

We now give semantics to our logic of total public announcements. Since we deal with an extension of $\mathbf{S} 5_{n}$, we will only need Kripke structures where the accessibility relations are equivalence relations.

Definition 3 An $n$-Kripke structure $\mathrm{K}=\left(S, R_{1}, \ldots, R_{n}, V\right)$ is an $(n+2)$ tuple, where $S \neq \emptyset$ is a set of states, $R_{i} \subseteq S^{2}$ for all $i \in \mathcal{A}$, and $V: \mathcal{P} \mapsto$ $\operatorname{Pow}(S)$ is a valuation function. 
Since $n$ is fixed, we will drop it and use only the term Kripke structure. The set $S$ is called the universe of $\mathrm{K}$, denoted by $|\mathrm{K}|$. In the sequel, we will write $\mathcal{K}_{n}^{e q}$ for the class of all Kripke structures with equivalence relations exclusively.

We will now define the validity of an $\mathcal{L}_{n}^{\mathrm{A}}$ formula in a state $s$ of a Kripke structure $\mathrm{K}$. The crucial point in this definition is the case of $[\alpha] \beta$, where we simultaneously define an operation on $\mathrm{K}$, depending on $\alpha$ and $s$.

Definition 4 Let $\mathrm{K}=\left(S, R_{1}, \ldots, R_{n}, V\right)$ be an arbitrary Kripke structure and $s \in S$ be given. The validity of $\mathcal{L}_{n}^{\mathrm{A}}$ formulas in the Kripke-world $\mathrm{K}, s$ is inductively defined as follows.

$$
\begin{array}{rll}
\mathrm{K}, s \models p & \text { iff } & s \in V(p), \\
\mathrm{K}, s=\neg \alpha & \text { iff } & \mathrm{K}, s \mid \models \alpha, \\
\mathrm{K}, s \models \alpha \wedge \beta & \text { iff } & \mathrm{K}, s \models \alpha \text { and } \mathrm{K}, s \models \beta, \\
\mathrm{K}, s \models K_{i} \alpha & \text { iff } & \text { for every } t \in S, s R_{i} t \Rightarrow \mathrm{K}, t \models \alpha, \\
\mathrm{K}, s \models[\alpha] \beta & \text { iff } & \mathrm{K}^{\alpha, s}, s \models \beta,
\end{array}
$$

where for given $\alpha \in \mathcal{L}_{n}^{\mathrm{A}}$ and $s \in|\mathrm{K}|$, the Kripke structure $\mathrm{K}^{\alpha, s}$ is simultaneously defined by

$$
\begin{aligned}
\mathrm{K}^{\alpha, s} & :=\left(S, R_{1}^{\alpha, s}, \ldots, R_{n}^{\alpha, s}, V\right), \\
R_{i}^{\alpha, s} & := \begin{cases}R_{i} \cap\left\{(u, v) \in S^{2} \mid \mathrm{K}, u \models \alpha \text { iff } \mathrm{K}, \mathrm{v} \models \alpha\right\} & \text { if } \mathrm{K}, s \models \alpha, \\
R_{i} & \text { if } \mathrm{K}, s \not \models \alpha .\end{cases}
\end{aligned}
$$

We say that an $\mathcal{L}_{n}^{\mathrm{A}}$ formula $\alpha$ is valid in the Kripke structure $\mathrm{K}(\mathrm{K} \models \alpha)$, iff for all $s \in S, \mathrm{~K}, s \models \alpha$. The formula $\alpha$ is valid with respect to $\mathcal{K}_{n}^{e q}\left(\mathcal{K}_{n}^{e q} \models \alpha\right)$, iff for all $\mathrm{K} \in \mathcal{K}_{n}^{e q}, \mathrm{~K}=\alpha$. Further, we say that $\alpha$ is satisfiable in $\mathcal{K}_{n}^{e q}$, iff there is a $\mathrm{K} \in \mathcal{K}_{n}^{e q}$ and an $s \in|\mathrm{K}|$, such that $\mathrm{K}, s \models \alpha$.

If all $R_{i}$ in $\mathrm{K}$ are equivalence relations, then $\mathrm{K}^{\alpha, s}$ belongs to $\mathcal{K}_{n}^{e q}$, as is stated in the next lemma. It is an immediate consequence of the definition of $R_{i}^{\alpha, s}$.

Lemma 5 For all Kripke structures $\mathrm{K}$, all $\mathcal{L}_{n}^{\mathrm{A}}$ formulas $\alpha$, and all $s \in|\mathrm{K}|$ we have

$$
\mathrm{K} \in \mathcal{K}_{n}^{e q} \quad \Rightarrow \quad \mathrm{K}^{\alpha, s} \in \mathcal{K}_{n}^{e q}
$$

Example 6 (Wise men puzzle) Alice, Bob, and Charlie wear a hat and cannot see its color. But they can see, of course, the color of the others' hats. There are only two red and three blue hats, and every person knows that. The Kripke structure K, which represents this situation is shown in Figure 1 (reflexivity and symmetry of the relations $R_{A}, R_{B}$, and $R_{C}$ are self-evident). 


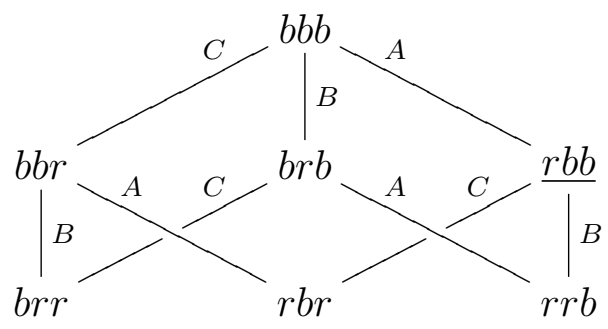

Figure 1: The initial structure

In the state $r b b$, Alice wears a red hat, whereas Bob and Charlie both wear a blue hat. Now, Alice publicly announces, that she does not know the color of her hat, which is true. After that, Bob announces the same true fact. This results in the Kripke structure

$$
\left(\mathrm{K}^{\left.\neg K_{A} r_{A} \wedge \neg K_{A} b_{A}, r b b\right) \neg K_{B} r_{B} \wedge \neg K_{B} b_{B}, r b b},\right.
$$

which is illustrated in Figure 2.

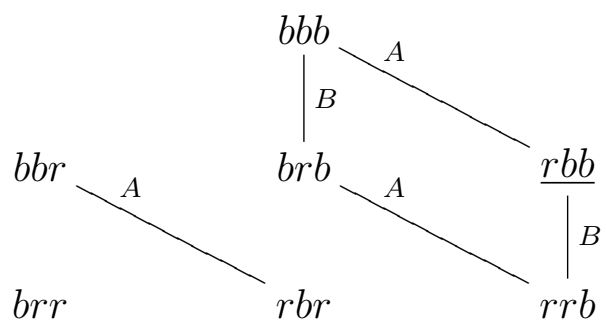

Figure 2: The situation after two announcements

Now Charlie knows that he is wearing a blue hat. Observe, that for a false announcement, e.g. $b_{A}$ in the state $r b b$, we have that $\mathrm{K}^{b_{A}, r b b}=\mathrm{K}$.

Soundness of our system can be proved in the usual way.

Lemma 7 The system $\mathrm{S}_{n}^{\mathrm{A}}$ is sound with respect to $\mathcal{K}_{n}^{e q}$, i.e. for all $\mathcal{L}_{n}^{\mathrm{A}}$ formulas $\alpha$ we have

$$
\mathrm{S}_{n}^{\mathrm{A}} \vdash \alpha \quad \Rightarrow \quad \mathcal{K}_{n}^{e q} \models \alpha .
$$

Proof The proof is by induction on the length of the derivation. In the base case, soundness of the axiom (A4) is proved as follows. Let $\mathrm{K} \in \mathcal{K}_{n}^{e q}, s \in|\mathrm{K}|$, 
and $i \in \mathcal{A}$ be given and assume that $\mathrm{K}, s \models \alpha$. Then we have

$$
\begin{array}{rll}
\mathrm{K}, s \models[\alpha] K_{i} \beta \quad & \text { iff } \quad \mathrm{K}^{\alpha, s}, s \models K_{i} \beta \\
& \text { iff } \quad \text { for all } t \in S, s R_{i}^{\alpha, s} t \Rightarrow \mathrm{K}^{\alpha, s}, t \models \beta \\
\text { iff } \quad \text { for all } t \in S, s R_{i} t \text { and } \mathrm{K}, t \models \alpha \Rightarrow \mathrm{K}, t \models[\alpha] \beta & \text { iff } \quad \mathrm{K}, s \models K_{i}(\alpha \rightarrow[\alpha] \beta) .
\end{array}
$$

In the induction step, soundness of the rule (NEC.2) immediately follows from Lemma 5.

Completeness of $\mathrm{S}_{n}^{\mathrm{A}}$ can be proved via a translation from $\mathcal{L}_{n}^{\mathrm{A}}$ to $\mathcal{L}_{n}$ since the two languages have the same expressive strength. As a preparation, we will define a translation from $\left\{[\alpha] \beta \mid \alpha, \beta \in \mathcal{L}_{n}\right\}$ to $\mathcal{L}_{n}$.

Definition 8 The function $h$ from $\left\{[\alpha] \beta \mid \alpha, \beta \in \mathcal{L}_{n}\right\}$ to $\mathcal{L}_{n}$ is inductively defined by

$$
\begin{aligned}
h([\alpha] p) & :=p \\
h([\alpha] \neg \beta) & :=\neg h([\alpha] \beta), \\
h([\alpha](\beta \wedge \gamma)) & :=h([\alpha] \beta) \wedge h([\alpha] \gamma), \\
h\left([\alpha] K_{i} \beta\right) & :=\left(\neg \alpha \wedge K_{i} \beta\right) \vee\left(\alpha \wedge K_{i}(\alpha \rightarrow h([\alpha] \beta))\right) .
\end{aligned}
$$

Of course, $h$ eliminates the announcement operator. Its definition leads to two important properties which we state in the following lemma.

Lemma 9 For all $\mathcal{L}_{n}^{\mathrm{A}}$ formulas $\alpha, \beta$ and all $\mathcal{L}_{n}$ formulas $\varphi, \psi$ we have

$$
\mathrm{S}_{n}^{\mathrm{A}} \vdash[\varphi] \psi \leftrightarrow h([\varphi] \psi) \quad \text { and } \quad \mathrm{S}_{n}^{\mathrm{A}} \vdash \alpha \leftrightarrow \beta \Rightarrow \mathrm{S}_{n}^{\mathrm{A}} \vdash[\alpha] \psi \leftrightarrow[\beta] \psi .
$$

We are now able to define our translation $t$, which eliminates the announcement operator in every $\mathcal{L}_{n}^{\mathrm{A}}$ formula.

Definition 10 The translation $t$ from $\mathcal{L}_{n}^{\mathrm{A}}$ to $\mathcal{L}_{n}$ is inductively defined by

$$
\begin{aligned}
t(p) & :=p, \\
t(\neg \alpha) & :=\neg t(\alpha), \\
t(\alpha \wedge \beta) & :=t(\alpha) \wedge t(\beta), \\
t\left(K_{i} \alpha\right) & :=K_{i} t(\alpha), \\
t([\alpha] \beta) & :=h([t(\alpha)] t(\beta)) .
\end{aligned}
$$

It is obvious that for every $\mathcal{L}_{n}^{\mathrm{A}}$ formula $\alpha$, its translation $t(\alpha)$ is a formula of $\mathcal{L}_{n}$. In addition, we can prove the equivalence of $\alpha$ and $t(\alpha)$ in $\mathrm{S}_{n}^{\mathrm{A}}$. 
Lemma 11 For all $\mathcal{L}_{n}^{\mathrm{A}}$ formulas $\alpha$ we have

$$
\mathrm{S}_{n}^{\mathrm{A}} \vdash \alpha \leftrightarrow t(\alpha) .
$$

Lemma 11 is very helpful for proofs by induction on arbitrary $\mathcal{L}_{n}^{\mathrm{A}}$ formulas. Making use of it, one can easily show the following property.

Corollary 12 For all $\mathcal{L}_{n}^{\mathrm{A}}$ formulas $\alpha, \beta$, and $\gamma$ we have

$$
\mathrm{S}_{n}^{\mathrm{A}} \vdash \alpha \leftrightarrow \beta \Rightarrow \mathrm{S}_{n}^{\mathrm{A}} \vdash[\alpha] \gamma \leftrightarrow[\beta] \gamma .
$$

As another consequence of Lemma 11 we get the following equivalence concerning consecutive announcement operators.

Lemma 13 For all $\mathcal{L}_{n}^{\mathrm{A}}$ formulas $\alpha, \beta$, and $\gamma$ we have

$$
\mathrm{S}_{n}^{\mathrm{A}} \vdash \alpha \wedge[\alpha] \beta \rightarrow([\alpha][\beta] \gamma \leftrightarrow[\alpha \wedge[\alpha] \beta] \gamma) .
$$

Making use of Lemma 11, we can easily show completeness of $\mathrm{S}_{n}^{\mathrm{A}}$.

Lemma 14 The system $\mathrm{S}_{n}^{\mathrm{A}}$ is complete with respect to $\mathcal{K}_{n}^{e q}$, i.e. for all $\mathcal{L}_{n}^{\mathrm{A}}$ formulas $\alpha$ we have

$$
\mathcal{K}_{n}^{e q} \models \alpha \quad \Rightarrow \quad \mathrm{S}_{n}^{\mathrm{A}} \vdash \alpha .
$$

Proof Assuming $\mathcal{K}_{n}^{e q} \models \alpha$, we get $\mathcal{K}_{n}^{e q} \models t(\alpha)$ by soundness and Lemma 11. Due to completeness of $\mathrm{S} 5_{n}$, we have $\mathrm{S} 5_{n} \vdash t(\alpha)$, which yields $\mathrm{S} 5_{n}^{\mathrm{A}} \vdash t(\alpha)$ because $\mathrm{S} 5_{n}$ is contained in $\mathrm{S} 5_{n}^{\mathrm{A}}$. Now, we get $\mathrm{S} 5_{n}^{\mathrm{A}} \vdash \alpha$ by Lemma 11 .

In a next step, we define announcement-resistant $\mathcal{L}_{n}^{\mathrm{A}}$ formulas. This notion is inspired by - but different than - the notion of successful formulas, see [8]. A formula $\alpha$ is successful if $[\alpha] \alpha$ is valid. However, in our setting not even propositions would be successful formulas. As an alternative, we introduce the class of announcement-resistant formulas.

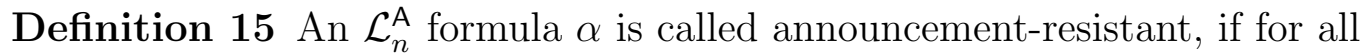
$\mathcal{L}_{n}^{\mathrm{A}}$ formulas $\beta$ we have

$$
\mathrm{S}_{n}^{\mathrm{A}} \vdash \alpha \rightarrow[\beta] \alpha .
$$

Observe, that $\mathrm{S}_{n}^{\mathrm{A}}$ proves $\alpha \rightarrow[\beta]^{k} \alpha$ for all $k \geq 0$, if $\alpha$ is announcementresistant. There are many announcement-resistant formulas, as the following lemma shows.

\section{Lemma 16}

1. All $\mathcal{L}_{0}$ formulas as well as all provable $\mathcal{L}_{n}^{\mathrm{A}}$ formulas are announcementresistant. 
2. If $\alpha$ and $\beta$ are announcement-resistant, then so also are the formulas $\alpha \wedge \beta, \alpha \vee \beta$, and $K_{i} \alpha$.

By the previous lemma, we know that $K_{i} \alpha$ is announcement-resistant for all $\mathcal{L}_{0}$ formulas $\alpha$. That means knowledge in propositional formulas can never be contracted by public announcements. We can therefore say that the logic of total public announcements formalizes expansion for propositional knowledge.

As we have seen in Example 6, agents can really expand their knowledge due to announcements. The next lemma shows that they learn true announcement-resistant formulas by one single announcement.

Lemma 17 Let $\alpha$ be an announcement-resistant $\mathcal{L}_{n}^{\mathrm{A}}$ formula. Then for all $k \geq 1$, all $m \geq 0$, and all $i_{1}, \ldots, i_{m} \in \mathcal{A}$ we have

$$
\mathrm{S}_{n}^{\mathrm{A}} \vdash \alpha \rightarrow[\alpha]^{k} K_{i_{m}} \ldots K_{i_{1}} \alpha .
$$

\section{Incorporating common knowledge}

Lemma 17 even shows that agents can acquire so-called common knowledge. In this section, we will extend our logic of total public announcements by common knowledge operators. To this aim we have to formalize the notion of mutual knowledge. For every non-empty group $G \subseteq \mathcal{A}$ of agents, the formula $\mathrm{E}_{G}^{k} \alpha$ is inductively defined by

$$
\mathrm{E}_{G}^{0} \alpha:=\alpha \quad \text { and } \quad \mathrm{E}_{G}^{k+1} \alpha:=\bigwedge_{i \in G}\left(K_{i} \mathrm{E}_{G}^{k} \alpha\right) .
$$

We simply write $\mathrm{E}_{G} \alpha$ for $\mathrm{E}_{G}^{1} \alpha$ to express that everybody in $G$ knows $\alpha$. The following property holds.

Lemma 18 For all $\mathcal{L}_{n}^{\mathrm{A}}$ formulas $\alpha$ and $\beta$ we have

$$
\mathrm{S}_{n}^{\mathrm{A}} \vdash \alpha \rightarrow\left([\alpha] \mathrm{E}_{G} \beta \leftrightarrow \mathrm{E}_{G}(\alpha \rightarrow[\alpha] \beta)\right) .
$$

The language $\mathcal{L}_{n}^{\mathrm{C}, \mathrm{A}}$ of common knowledge and public announcements is the language $\mathcal{L}_{n}^{\mathrm{A}}$ expanded by the common knowledge operator $\mathrm{C}_{G}$ for every nonempty group $G \subseteq \mathcal{A}$ of agents. To define the validity of $\mathcal{L}_{n}^{\mathrm{C}, \mathrm{A}}$ formulas in a Kripke-world $\mathrm{K}, s$, we add the following clause to Definition 4.

$$
\mathrm{K}, s \models \mathrm{C}_{G} \alpha \quad \text { iff } \quad \text { for every } t \in|\mathrm{K}|, s\left(R_{G}\right)^{\star} t \Rightarrow \mathrm{K}, t \models \alpha \text {, }
$$


where $\left(R_{G}\right)^{\star}$ denotes the transitive closure of $R_{G}:=\bigcup\left\{R_{i} \mid i \in G\right\}$, see Fagin et al. [11]. It is an easy exercise to show that

$$
\mathrm{K}, s \models \mathrm{C}_{G} \alpha \quad \text { iff } \quad \text { for every } t \text {, there is a } G \text {-path from s to } \mathrm{t} \Rightarrow \mathrm{K}, t=\alpha \text {. }
$$

The system $\mathrm{S} 5_{n}^{\mathrm{C}, \mathrm{A}}$ is defined as extension of $\mathrm{S} 5_{n}^{\mathrm{A}}$ by an additional announcement axiom, the axioms and rules for common knowledge, as well as a rule for common knowledge after an announcement.

Definition 19 The theory $\mathrm{S} 5_{n}^{\mathrm{C}, \mathrm{A}}$ is defined to be $\mathrm{S} 5_{n}^{\mathrm{A}}$ augmented by the following axioms and rules.

$$
\begin{aligned}
& \text { (A6) } \quad \alpha \wedge[\alpha] \beta \rightarrow([\alpha][\beta] \gamma \leftrightarrow[\alpha \wedge[\alpha] \beta] \gamma), \\
& \text { (C) } \quad \mathrm{C}_{G} \alpha \rightarrow \mathrm{E}_{G}\left(\alpha \wedge \mathrm{C}_{G} \alpha\right), \\
& \left(\text { IND.1) } \frac{\alpha \rightarrow \mathrm{E}_{G}(\alpha \wedge \beta)}{\alpha \rightarrow \mathrm{C}_{G} \beta}, \quad\left(\text { IND.2) } \frac{\alpha \rightarrow[\beta] \gamma \quad \alpha \wedge \beta \rightarrow \mathrm{E}_{G}(\beta \rightarrow \alpha)}{\alpha \wedge \beta \rightarrow[\beta] \mathrm{C}_{G} \gamma} .\right.\right.
\end{aligned}
$$

Axiom (A6) is provable in $\mathrm{S} 5_{n}^{\mathrm{A}}$, see Lemma 13. However, this is not the case for $\mathrm{S} 5_{n}^{\mathrm{C}, \mathrm{A}}$ since there is no translation available that eliminates the announcement operators. The rule (IND.2) is a slight modification of the action rule from Baltag et al. in [1, 2].

To show soundness and completeness, we will need the following notion of a $G^{\alpha}$-path.

Definition 20 Let $\alpha \in \mathcal{L}_{n}^{\mathrm{C}, \mathrm{A}}, \mathrm{K} \in \mathcal{K}_{n}^{e q}, \emptyset \neq G \subseteq \mathcal{A}$, and $s, t \in|\mathrm{K}|$. Then we say that there is a $G^{\alpha}$-path from $s$ to $t$, if there are states $u_{1}, u_{2}, \ldots, u_{k} \in|\mathrm{K}|$ s.t. $s R_{G} u_{1} R_{G} u_{2} R_{G} \ldots R_{G} u_{k}, u_{k}=t$, and for all $j, 1 \leq j \leq k \Rightarrow \mathrm{K}, u_{j}=\alpha$.

It is easy to see that $\mathrm{K}, s=\alpha$ implies

$$
\begin{aligned}
& \mathrm{K}, s \models[\alpha] \mathrm{C}_{G} \beta \quad \text { iff } \\
& \quad \text { for every } t \text {, there is a } G^{\alpha} \text {-path from } s \text { to } t \Rightarrow \mathrm{K}, t=[\alpha] \beta .
\end{aligned}
$$

Lemma 21 The system $\mathrm{S} 5_{n}^{\mathrm{C}, \mathrm{A}}$ is sound with respect to $\mathcal{K}_{n}^{e q}$, i.e. for all $\mathcal{L}_{n}^{\mathrm{C}, \mathrm{A}}$ formulas $\alpha$ we have

$$
\mathrm{S} 5_{n}^{\mathrm{C}, \mathrm{A}} \vdash \alpha \Rightarrow \mathcal{K}_{n}^{e q} \models \alpha .
$$

Proof The proof is by induction on the length of the derivation. In the induction step, soundness of the rule (IND.2) is proved as follows. Suppose, the formula $\alpha \wedge \beta \rightarrow[\beta] C_{G} \gamma$ has been derived with the rule (IND.2). Then, by induction hypothesis, we know that the formulas (a) $\alpha \rightarrow[\beta] \gamma$ and (b) $\alpha \wedge \beta \rightarrow \mathrm{E}_{G}(\beta \rightarrow \alpha)$ are valid. Now, take any Kripke-world $\mathrm{K}, s$ such that 
$\mathrm{K}, s \models \alpha \wedge \beta$. Using (b) we get that $\mathrm{K}, t \models \alpha$ in every world $t \in|\mathrm{K}|$ which is reachable on a $G^{\beta}$-path from $s$. But then, by (a), we have that $\mathrm{K}, t \models[\beta] \gamma$ in every $t$ reachable on a $G^{\beta}$-path from $s$. This implies, by definition of the announcement semantics, that $\mathrm{K}, s=[\beta] \mathrm{C}_{G} \gamma$, and we are done.

Lemma 22 For all $\mathcal{L}_{n}^{\mathrm{C}, \mathrm{A}}$ formulas $\alpha, \beta$, and $\gamma$ we have

$$
\begin{aligned}
& \mathrm{S}_{n}^{\mathrm{C}, \mathrm{A}} \vdash \alpha \wedge \mathrm{C}_{G}(\alpha \rightarrow[\alpha] \beta) \rightarrow[\alpha] \mathrm{C}_{G} \beta, \\
& \mathrm{S}_{n}^{\mathrm{C}, \mathrm{A}} \vdash \alpha \leftrightarrow \beta \Rightarrow \mathrm{S}_{n}^{\mathrm{C}, \mathrm{A}} \vdash[\alpha] \gamma \leftrightarrow[\beta] \gamma .
\end{aligned}
$$

Since the formula $\alpha \wedge[\alpha] C_{G} \beta \rightarrow \mathrm{C}_{G}(\alpha \rightarrow[\alpha] \beta)$ is not valid (compare with (A4)), there is no translation available that could be used to give an easy completeness proof of $\mathrm{S}_{n}^{\mathrm{C}, \mathrm{A}}$. Thus we have to employ maximal consistent sets to show completeness. Our argument is the same as the one presented in [11] for the logic of common knowledge except that we have more cases in the truth lemma. We start by defining the closure $\operatorname{cl}(\alpha)$ of a formula $\alpha$.

Definition 23 For all $\mathcal{L}_{n}^{\mathrm{C}, \mathrm{A}}$ formulas $\alpha, s u b^{+}(\alpha)$ is the smallest set which satisfies the following conditions.

1. $\alpha \in \operatorname{sub}^{+}(\alpha)$.

2. If $\neg \beta \in \operatorname{sub}^{+}(\alpha)$, then $\beta \in \operatorname{sub}^{+}(\alpha)$.

3. If $\beta \wedge \gamma \in \operatorname{sub}^{+}(\alpha)$, then $\beta, \gamma \in \operatorname{sub}^{+}(\alpha)$.

4. If $K_{i} \beta \in \operatorname{sub}^{+}(\alpha)$, then $\beta \in s u b^{+}(\alpha)$.

5. If $\mathrm{C}_{G} \beta \in s u b^{+}(\alpha)$, then $\mathrm{E}_{G} \beta, \mathrm{E}_{G} \mathrm{C}_{G} \beta \in s u b^{+}(\alpha)$.

6. If $[\beta] p \in s u b^{+}(\alpha)$, then $\beta, p \in s u b^{+}(\alpha)$.

7. If $[\beta] \neg \gamma \in \operatorname{sub}^{+}(\alpha)$, then $[\beta] \gamma, \neg \gamma \in \operatorname{sub}^{+}(\alpha)$.

8. If $[\beta](\gamma \wedge \delta) \in \operatorname{sub}^{+}(\alpha)$, then $[\beta] \gamma,[\beta] \delta, \gamma \wedge \delta \in \operatorname{sub}^{+}(\alpha)$.

9. If $[\beta] K_{i} \gamma \in s u b^{+}(\alpha)$, then $K_{i} \gamma, K_{i}(\beta \rightarrow[\beta] \gamma) \in \operatorname{sub}^{+}(\alpha)$.

10. If $[\beta] \mathrm{C}_{G} \gamma \in \operatorname{sub}^{+}(\alpha)$, then $\mathrm{C}_{G} \gamma, \mathrm{E}_{G}\left(\beta \rightarrow[\beta] \mathrm{C}_{G} \gamma\right) \in s u b^{+}(\alpha)$.

11. If $[\beta][\gamma] \delta \in \operatorname{sub}^{+}(\alpha)$, then $[\beta] \delta,[\gamma] \delta,[\beta \wedge[\beta] \gamma] \delta \in s u b^{+}(\alpha)$.

The closure of $\alpha$ is defined by $\operatorname{cl}(\alpha):=\operatorname{sub}^{+}(\alpha) \cup\left\{\neg \beta \mid \beta \in \operatorname{sub}^{+}(\alpha)\right\}$.

Observe, that for $\alpha=[\beta] \gamma$, we immediately get both $s u b^{+}(\beta) \subseteq s u b^{+}(\alpha)$ and $s u b^{+}(\gamma) \subseteq s u b^{+}(\alpha)$. 
Lemma 24 For every $\mathcal{L}_{n}^{\mathrm{C}, \mathrm{A}}$ formula $\alpha$, the set $\operatorname{cl}(\alpha)$ is finite.

Note that $\operatorname{cl}(\alpha)$ is not closed under complements: for a given $\beta \in \operatorname{cl}(\alpha)$ we need not have $\neg \beta \in \operatorname{cl}(\alpha)$. However, there is always a formula $\sim \beta \in \operatorname{cl}(\alpha)$, which is equivalent to $\neg \beta$.

Definition 25 For every $\mathcal{L}_{n}^{\mathrm{C}, \mathrm{A}}$ formula $\alpha$, the canonical structure of $\alpha$ is defined by $\mathrm{K}_{\alpha}=\left(\operatorname{con}(\alpha), R_{1}, \ldots, R_{n}, V\right)$, where

$$
\begin{aligned}
\operatorname{con}(\alpha) & :=\left\{U \cap \operatorname{cl}(\alpha) \mid U \text { is a maximal } \mathrm{S}_{n}^{\mathrm{C}, \mathrm{A}} \text {-consistent set }\right\} \\
R_{i} & :=\left\{(X, Y) \mid X / K_{i}=Y / K_{i}\right\} \\
V(p) & :=\{X \mid p \in X\}
\end{aligned}
$$

and $X / K_{i}$ denotes the set $\left\{\beta \mid K_{i} \beta \in X\right\}$.

For the notion of a maximal consistent set with respect to a theory, see for instance [11]. Observe, that $\mathrm{K}_{\alpha}$ is in $\mathcal{K}_{n}^{e q}$ for all $\mathcal{L}_{n}^{\mathrm{C}, \mathrm{A}}$ formulas $\alpha$.

Lemma 26 (Truth Lemma) Let $\alpha$ be an arbitrary $\mathcal{L}_{n}^{\mathrm{C}, \mathrm{A}}$ formula and $\mathrm{K}_{\alpha}$ be its canonical structure. Then we have for all $\beta \in \operatorname{cl}(\alpha)$ and all $X \in \operatorname{con}(\alpha)$,

$$
\beta \in X \quad \Leftrightarrow \quad \mathrm{K}_{\alpha}, X \models \beta \text {. }
$$

Proof We prove this lemma by induction on $\beta$, and we omit the cases where $\beta$ does not begin with an announcement operator because they are standard. Hence assume $\beta$ begins with an announcement operator. We show by side induction on $\delta$ that for any announcement operator $[\gamma]$ the claim holds for $\beta=[\gamma] \delta$. The base case is immediate, we have $[\gamma] p \in X$ iff $\mathrm{K}_{\alpha}, X \models[\gamma] p$ by axiom (A1) and Definition 25. The cases $\beta=[\gamma] \neg \varphi$ and $\beta=[\gamma](\varphi \wedge \psi)$ are also straightforward.

Now let $\beta=[\gamma] K_{i} \varphi$. The interesting step is the case $\mathrm{K}_{\alpha}, X \models \gamma$ in the direction from right to left. First, one can show that the set

$$
Y:=\left\{K_{i} \xi \mid K_{i} \xi \in X\right\} \cup\left\{\neg K_{i} \xi \mid \neg K_{i} \xi \in X\right\} \cup\{\gamma, \neg[\gamma] \varphi\}
$$

is inconsistent, using the induction hypothesis for $\gamma$ and $[\gamma] \varphi$. Using the axioms ( $\mathrm{T}),(4)$, and (5), we can prove the existence of a set

$$
Z:=\left\{K_{i} \xi_{1}, \ldots, K_{i} \xi_{k}, \neg K_{i} \xi_{k+1}, \ldots, \neg K_{i} \xi_{l}\right\} \subseteq Y
$$

s.t. the set $Z \cup\left\{\neg K_{i}(\gamma \rightarrow[\gamma] \varphi)\right\}$ is inconsistent. Since $Z \subseteq X$ and $X \in$ $\operatorname{con}(\alpha)$, we have $K_{i}(\gamma \rightarrow[\gamma] \varphi) \in X$. Using axiom (A4) and again the induction hypothesis for $\gamma$ we get that $[\gamma] K_{i} \varphi \in X$. 
For $\beta=[\gamma] \mathrm{C}_{G} \varphi$, the case $\mathrm{K}_{\alpha}, X \models \gamma$ in the direction from right to left is the challenging part of the proof. For every $Y \in \operatorname{con}(\alpha)$ and for the set $\mathcal{B}:=\left\{Z \in \operatorname{con}(\alpha) \mid \mathrm{K}_{\alpha}, Z \models[\gamma] \mathrm{C}_{G} \varphi\right\}$, we define the formulas

$$
\psi_{Y}:=\bigwedge_{\xi \in Y} \xi \quad \text { and } \quad \chi_{\mathcal{B}}:=\bigvee_{Y \in \mathcal{B}} \psi_{Y}
$$

It is not hard to show, that the formulas $\chi_{\mathcal{B}} \rightarrow[\gamma] \varphi$ and $\chi_{\mathcal{B}} \wedge \gamma \rightarrow \mathrm{E}_{G}\left(\gamma \rightarrow \chi_{\mathcal{B}}\right)$ are both derivable. Applying the rule (IND.2) we get $\vdash \chi_{\mathcal{B}} \wedge \gamma \rightarrow[\gamma] \mathrm{C}_{G} \varphi$. Since $X \in \mathcal{B}$ by assumption, and $\gamma \in X$ by induction hypothesis, we have $\vdash \psi_{X} \rightarrow[\gamma] C_{G} \varphi$. Hence, we know that $[\gamma] C_{G} \varphi \in X$.

The last case of our induction is $\beta=[\gamma][\varphi] \psi$. Here we distinct the three cases $\sim \gamma \in X, \sim[\gamma] \varphi \in X$, and $\gamma,[\gamma] \varphi \in X$. All three cases are straightforward using the axioms (A5) and (A6), respectively. Observe, that we need the second induction hypothesis in the third case.

Due to the Truth Lemma, we know that every $\mathrm{S} 5{ }_{n}^{\mathrm{C}, \mathrm{A}}$-consistent formula is satisfiable in $\mathcal{K}_{n}^{e q}$, thus we have completeness.

Theorem 27 The system $\mathrm{S}_{n}^{\mathrm{C}, \mathrm{A}}$ is complete with respect to $\mathcal{K}_{n}^{\text {eq }}$, i.e. for all $\mathcal{L}_{n}^{\mathrm{C}, \mathrm{A}}$ formulas $\alpha$ we have

$$
\mathcal{K}_{n}^{e q} \models \alpha \quad \Rightarrow \quad \mathrm{S} 5_{n}^{\mathrm{C}, \mathrm{A}} \vdash \alpha .
$$

As an immediate consequence of our completeness proof, we get the finite model property and that the satisfiability problem is decidable.

We now extend the definition of announcement-resistance to the richer language. An $\mathcal{L}_{n}^{\mathrm{C}, \mathrm{A}}$ formula $\alpha$ is called announcement-resistant, if

$$
\mathrm{S}_{n}^{\mathrm{C}, \mathrm{A}} \vdash \alpha \rightarrow[\beta] \alpha
$$

for every $\mathcal{L}_{n}^{\mathrm{C}, \mathrm{A}}$ formula $\beta$.

It is still true, that all $\mathcal{L}_{0}$ formulas as well as all provable $\mathcal{L}_{n}^{\mathrm{C}, \mathrm{A}}$ formulas are announcement-resistant. In addition, if $\alpha$ and $\beta$ are announcement-resistant, then so also are the formulas $\alpha \wedge \beta, \alpha \vee \beta, K_{i} \alpha$, and $\mathrm{C}_{G} \alpha$.

Lemma 28 Let $\alpha$ be an announcement-resistant $\mathcal{L}_{n}^{\mathrm{C}, \mathrm{A}}$ formula and $G \subseteq \mathcal{A}$ be a non-empty group of agents. Then for all $k \geq 1$ we have

$$
\mathrm{S}_{n}^{\mathrm{C}, \mathrm{A}} \vdash \alpha \rightarrow[\alpha]^{k} \mathrm{C}_{G} \alpha .
$$




\section{Relativized common knowledge}

In this section, we will study operators for relativized common knowledge. We will present a sound and complete axiomatization for the logic of relativized common knowledge augmented by total public announcements.

The notion of relativized common knowledge is a generalized version of common knowledge. The language of $\mathcal{L}_{n}^{\mathrm{RC}, \mathrm{A}}$ formulas is the language $\mathcal{L}_{n}^{\mathrm{A}}$ extended by the binary operator $\mathrm{RC}_{G}$ for every non-empty group $G \subseteq \mathcal{A}$ of agents. The validity for relativized common knowledge is defined by

$$
\begin{aligned}
\mathrm{K}, s \models \mathrm{RC}_{G}(\alpha, \beta) & \text { iff } \\
& \text { for every } t \text {, there is a } G^{\alpha} \text {-path from } s \text { to } t \Rightarrow \mathrm{K}, t \models \beta .
\end{aligned}
$$

It is obvious that common knowledge can be defined by means of relativized common knowledge via $C_{G} \alpha:=\mathrm{RC}_{G}(\top, \alpha)$. It can be shown that relativized common knowledge is strictly more expressive than common knowledge, see van Benthem et al. [4, 5] for a proof. For instance, the until operator of temporal logic can be defined using the relativized common knowledge operator.

Definition 29 The theory $\mathrm{S} 5_{n}^{\mathrm{RC}, \mathrm{A}}$ is defined to be $\mathrm{S} 5_{n}^{\mathrm{A}}$ augmented by the following axioms and rules.

$$
\begin{aligned}
\alpha \rightarrow\left([\alpha] \mathrm{RC}_{G}(\beta, \gamma) \leftrightarrow \mathrm{RC}_{G}(\alpha \wedge[\alpha] \beta,[\alpha] \gamma)\right) \\
\mathrm{RC}_{G}(\alpha, \beta) \rightarrow \mathrm{E}_{G}\left(\alpha \rightarrow \beta \wedge \mathrm{RC}_{G}(\alpha, \beta)\right) \\
\left(\text { IND.3) } \frac{\alpha \rightarrow \mathrm{E}_{G}(\beta \rightarrow \alpha \wedge \gamma)}{\alpha \rightarrow \mathrm{RC}_{G}(\beta, \gamma)}\right.
\end{aligned}
$$

Lemma 30 For all $\mathcal{L}_{n}^{\mathrm{RC}, \mathrm{A}}$ formulas $\alpha, \beta$, and $\gamma$ we have

$$
\begin{aligned}
& \mathrm{S}_{n}^{\mathrm{RC}, \mathrm{A}} \vdash \mathrm{RC}_{G}(\alpha, \beta) \rightarrow(\alpha \rightarrow \beta), \\
& \mathrm{S}_{n}^{\mathrm{RC}, \mathrm{A}} \vdash \mathrm{RC}_{G}(\alpha, \beta \rightarrow \gamma) \rightarrow\left(\mathrm{RC}_{G}(\alpha, \beta) \rightarrow \mathrm{RC}_{G}(\alpha, \gamma)\right), \\
& \mathrm{S}_{n}^{\mathrm{RC}, \mathrm{A}} \vdash \mathrm{E}_{G}\left(\alpha \rightarrow \beta \wedge \mathrm{RC}_{G}(\alpha, \beta)\right) \rightarrow \mathrm{RC}_{G}(\alpha, \beta), \\
& \mathrm{S}_{n}^{\mathrm{RC}, \mathrm{A}} \vdash \alpha \Rightarrow \mathrm{S}_{n}^{\mathrm{RC}, \mathrm{A}} \vdash \mathrm{RC}_{G}(\beta, \alpha) .
\end{aligned}
$$

Theorem 31 The system $\mathrm{S} 5_{n}^{\mathrm{RC}, \mathrm{A}}$ is sound and complete with respect to $\mathcal{K}_{n}^{e q}$, i.e. for all $\mathcal{L}_{n}^{\mathrm{RC}, \mathrm{A}}$ formulas $\alpha$ we have

$$
\mathrm{S}_{n}^{\mathrm{RC}, \mathrm{A}} \vdash \alpha \quad \Leftrightarrow \quad \mathcal{K}_{n}^{e q} \models \alpha .
$$


Proof Soundness is proved as usual. The completeness proof for relativized common knowledge without announcement operators is similar to the proof of Theorem 27. The full system $\mathrm{S} 5_{n}^{\mathrm{RC}, \mathrm{A}}$ can now be treated by providing a translation which eliminates the announcements. Such a translation can easily be defined: simply observe that the axioms (A5) and (A7) imply

$$
\mathrm{S} 5_{n}^{\mathrm{RC}, \mathrm{A}} \vdash[\alpha] \mathrm{RC}_{G}(\beta, \gamma) \leftrightarrow\left(\neg \alpha \wedge \mathrm{RC}_{G}(\beta, \gamma)\right) \vee\left(\alpha \wedge \mathrm{RC}_{G}(\alpha \wedge[\alpha] \beta,[\alpha] \gamma)\right)
$$

(compare with the case for $[\alpha] K_{i} \beta$ in Lemma 2). Making use of that translation and of the completeness for the system without announcements, we can show completeness of $\mathrm{S}_{n}^{\mathrm{RC}, \mathrm{A}}$ as in Lemma 14.

\section{Discussion}

In the classical setting, public announcements are considered to be truthful. Thus, a specific announcement cannot happen in all possible worlds. If an announcement $\alpha$ is false in a given world, then the corresponding update action $[\alpha]$ cannot be performed, formally $[\alpha] \perp$ holds in that world.

We have presented a semantics, and corresponding formal systems, in which an announcement can happen in every possible world. Thus, announcements are total, that is the formula $\neg[\alpha] \perp$ is valid.

If an announcement is true, then an agent's knowledge gets updated; if it is false, then the agent's knowledge remains unchanged. In the classical setting, a false announcement cannot happen. In our approach it can happen but does not change an agent's knowledge. Thus we have a semantics which internalizes the idea of doing nothing when a false announcement is made.

This is a step towards a system in which an agent's knowledge and beliefs are considered. In such a setting, every announcement has to be performed since it may change an agent's beliefs even if its knowledge will not be affected. Thus it is necessary that announcements are total if we talk about both knowledge and beliefs of an agent.

In the present approach we use an alethic criterion (namely wether an announcement is true or false) to decide wether an agent's knowledge should be updated or not. Of course, one may argue about such a criterion. However, since an agent's knowledge has to be true by the knowledge axiom ( $\mathrm{T})$, it should only be updated by announcements that are indeed true. Therefore, unlike in the case of belief updates, it is not possible to use an epistemic criterion. The approach from [15], for instance, would lead to false knowledge by already accepting an announcement if it is consistent with the previous knowledge. 
There are already languages available in which our form of announcements can be expressed. However, they are very expressive: non-deterministic choice, truthful public announcements, and PDL-like test operators [1] are needed in order to model our system. We have presented a lean and direct syntax and semantics for total public announcements which leads to new concepts such as announcement-resistant formulas. An interesting open question is to give a syntactic characterization of this class of formulas. As already mentioned, further work will also be done to investigate systems for updating knowledge and beliefs of an agent.

\section{Acknowledgement}

We would like to thank the anonymous referees for the long list of comments which helped to improve the paper.

\section{References}

[1] Baltag, A., And Moss, L. S. Logics for epistemic programs. Synthese 139, 2 (2004), 165-224.

[2] Baltag, A., Moss, L. S., And Solecki, S. The logic of public announcements, common knowledge, and private suspicions. In TARK '98: Proceedings of the 7th conference on Theoretical aspects of rationality and knowledge (1998), Morgan Kaufmann Publishers, pp. 43-56.

[3] VAn Benthem, J. One is a lonely number: on the logic of communication. In Logic Colloquium '02 (2006), Z. Chatzidakis, P. Koepke, and W. Pohlers, Eds., no. 27 in Lecture Notes in Logic, ASL and A. K. Peters, pp. 96-129.

[4] van Benthem, J., van Eijck, J., And Kooi, B. Common knowledge in update logics. In TARK '05: Proceedings of the 10th conference on Theoretical aspects of rationality and knowledge (2005), National University of Singapore, pp. 253-261.

[5] van Benthem, J., van Eijck, J., And Kooi, B. Logics of communication and change. Information and Computation 204, 11 (2006), $1620-1662$.

[6] VAn Benthem, J., And Kooi, B. Reduction axioms for epistemic actions. In AiML '04: Proceedings of Advances in Modal Logic 5 (2004), 
R. Schmidt, I. Pratt-Hartmann, M. Reynolds, and H. Wansing, Eds., no. UMCS-04-9-1 in Technical Report Series, University of Manchester, pp. 197-211.

[7] VAn Ditmarsch, H. Knowledge games. PhD thesis, University of Groningen, 2000. ILLC Dissertation Series 2000-06.

[8] Van Ditmarsch, H. The russian cards problem. Studia Logica 75, 4 (2003), 31-62.

[9] van Ditmarsch, H., van der Hoek, W., and Kooi, B. Dynamic epistemic logic, vol. 337 of Synthese Library. Springer, 2007.

[10] van Ditmarsch, H., And Kooi, B. The secret of my success. Synthese 151, 2 (2005), 202-232.

[11] Fagin, R., Halpern, J. Y., Moses, Y., and Vardi, M. Y. Reasoning about Knowledge. MIT Press, 1995.

[12] Gerbrandy, J., And Groeneveld, W. Reasoning about information change. Journal of Logic, Language and Information 6, 2 (1997), $147-169$.

[13] PlazA, J. A. Logics of public communications. In Proceedings of the 4th International Symposium on Methodologies for Intelligent Systems (1989), M. Emrich, M. Pfeifer, M. Hadzikadic, and Z. Ras, Eds., pp. 201216.

[14] Renne, B. Bisimulation and public announcements in logics of evidence-based knowledge. In ESSLLI '06: Proceedings of the European Summer School in Logic, Language and Information, Workshop on Rationality and Knowledge (2006), S. Artemov and R. Parikh, Eds., Association for Logic, Language and Information, pp. 112-123.

[15] Steiner, D. A system for consistency preserving belief change. In ESSLLI '06: Proceedings of the European Summer School in Logic, Language and Information, Workshop on Rationality and Knowledge (2006), S. Artemov and R. Parikh, Eds., Association for Logic, Language and Information, pp. 133-144. 